\section{A Polarizing Microscope for Mapping Birefringent Objects in 3D Space}

\author{
Michael Shribak and Rudolf Oldenbourg \\ Marine Biological Laboratory, Woods Hole \\ mshribak@mbl.edu
}

The traditional polarizing microscope equipped with crossed linear polarizers shows a picture in which the image contrast of a birefringent object depends on both the amount of relative retardation induced by the object and the orientation of its principal axis. For example, if the orientation of the principal axis is parallel to one of the polarizers, then the birefringent object becomes invisible. For best visibility, the principal axis must be oriented at $45^{\circ}$ to the polarizers. In addition, the image intensity of the object is proportional to the square of the sine of the relative phase shit between the ordinary and the extraordinary ray passing through the object. The addition of a waveplate as compensator to the optical train provides the opportunity to improve the contrast and measure the relative retardation, but the principal restrictions of orientation dependence and non-linear image contrast remains.

The advent of new electro-optical devices, electronic imaging, and digital image processing have made possible the introduction of techniques that independently measure the relative retardation (also called retardance) and the orientation (also called azimuth) of birefringent objects. Measurement results are simultaneously obtained for all birefringent objects in the field of view and are displayed as separate images, one is called the retardance image and the other is called the azimuth image. These new types of polarizing microscopes typically incorporate electronically adjustable polarizers and waveplates, and a video camera with a digital image processing system and custom designed software. For example, optical systems have been reported that use either fixed linear polarizers and variable liquid crystal waveplates [1], or a rotating linear polarizer and fixed circular polarizers [2] In either case, a combination of adjustable polarizers and waveplates are used to create a sequence of polarization states for illuminating the specimen and for analyzing the light that is transmitted or reflected by the specimen. In quick succession, several raw images of the specimen are recorded at predetermined settings of the polarizers and waveplates and the images are processed in order to calculate the retardance and azimuth images of the specimen [3]. Instead of generating the raw images sequentially, it is also possible to generate them in parallel by using a non-polarizing beamsplitter with a set of polarization analyzers in the imaging path [4].

The advanced techniques are successfully used in industrial, research, and clinical applications, including studies of the cell cytoskeleton [5] and for in vitro fertilization procedures [6]. However, the retardance measured with these techniques still depends on the angle between the crystal optic axis of the birefringent object and the axis of the beam illuminating the object. If these two axes are nearly parallel to each other, the measured retardance value decreases dramatically and becomes zero for parallel axes. In principle, in order to change this angle it is possible to use a universal stage, originally invented by E.S. Fedorov more than 100 years ago [7]. With this apparatus the specimen, supported between two glass hemispheres, may be manually rotated through measured angles about vertical and horizontal axes. Hence, the specimen can be rotated to a position where the optic axis of the birefringent object under observation is perpendicular to the illumination beam and the maximum retardance is measured. The universal stage is relatively bulky and can accommodate only long working distance, and low resolution optics. While it allows the measurement of precise angles and the maximum retardance of an object it can do so only for relatively large objects, one object at a time. Therefore the universal stage has found use mainly in geological applications for the analysis of thin rock sections containing birefringent crystals that are at least a few microns in diameter.

We are now introducing a new technique that rotates the illumination beam instead of the specimen, combining in one instrument high numerical aperture optics, an aperture scanning device, and a universal compensator for measuring specimen birefringence with high sensitivity and high resolution. The so-called Scanned Aperture Pol-Scope is able to measure the maximum retardance of a uniaxial birefringent object, regardless of how it is oriented, and it can determine the two angles that characterize the orientation of the object in 3-dimensional space. In addition, the new technique measures all these parameters for every resolved specimen point simultaneously, requiring only a few seconds for measuring up to a million specimen points

A schematic of the Scanned Aperture Pol-Scope is shown in Fig. 1. The optical set-up includes a custom-made aperture scanning apparatus built with liquid crystal devices. The scanner implements two independent functions, an amplitude mask and a universal compensator, each of which is computer controlled. The amplitude mask, with 8 pie-shaped sectors, is used to partially block the aperture and steer the cone of light illuminating the specimen. The sectored universal compensator is used as both a polarization rectifier [8] and a birefringence measurement device [3]. Al components of the scanning device are bonded together and form a $7 \mathrm{~mm}$ thick optical flat with an approximate diameter of $25 \mathrm{~mm}$. The liquid crystal based scanning device was designed in collaboration with Cambridge Research and Instrumentation, Woburn, MA, who manufactured the device and its electronic control circuitry. The computer software for hardware control and image analysis was developed in-house.

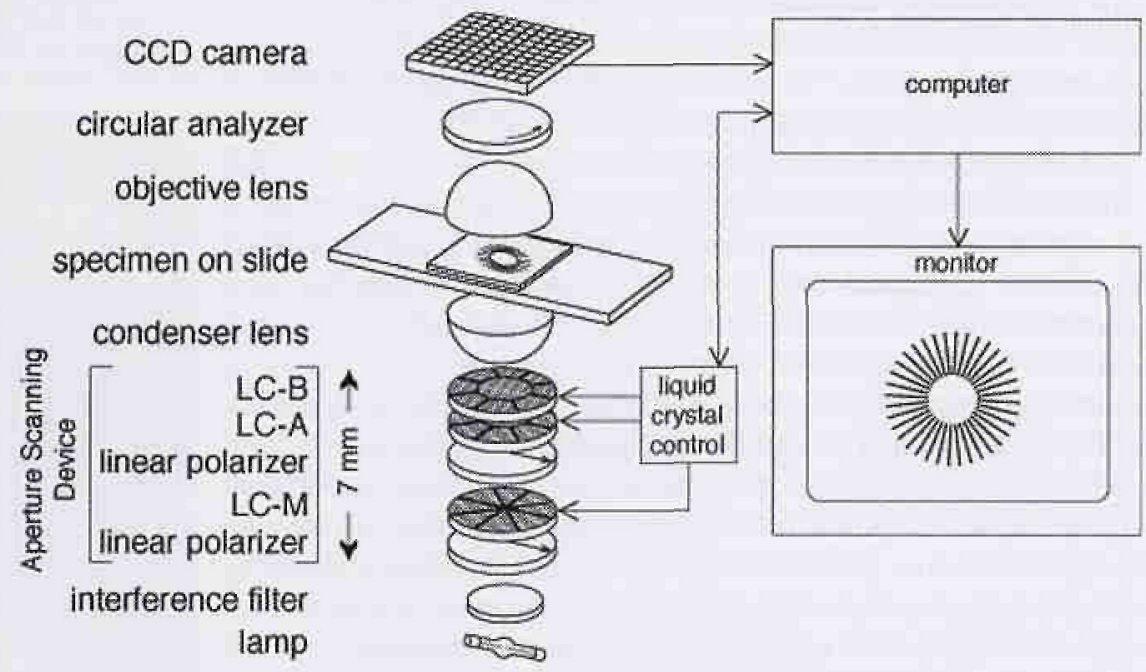

Fig. 1. Schematic of optical (left) and electronic components of the Scanned Aperture Pol-Scope. The optical set-up is based on a traditional light microscope enhanced by an aperture scanning device and an analyzer for circular polarized light. The scanning device includes the liquid crystal devices $L C-A, L C-B$, and LC-M. All liquid crystal devices are variable linear retarder plates with 8 or 9 individually controlled sectors. LC-M is sandwiched between two linear polarizers and is used to block or transmit light passing through 8 pie-shaped sectors. A pair of sectors in LC-A and LC-B arranged in series, function as a universal compensator for controlling the polarization state of the passing light. The computer controlled scanning device is placed in the front aperture plane of the condenser lens.

Before we discuss the operation of the instrument, we want to clarify the birefringence parameters it can measure. Let's assume we focus the instrument on a small, uniaxial, birefringent object such as a microtubule (MT), a rod-like biopolymer that has $25 \mathrm{~nm}$ diameter and can be several micron long (Fig. 2). The birefringence of microtubules, while small, can be measured with a polarizing microscope [9]. The optic axis of a microtubule is parallel to the rod-axis and the maximum retardance induced by a single MT is less than one tenth of a nanometer. A dense array of parallel MTs 


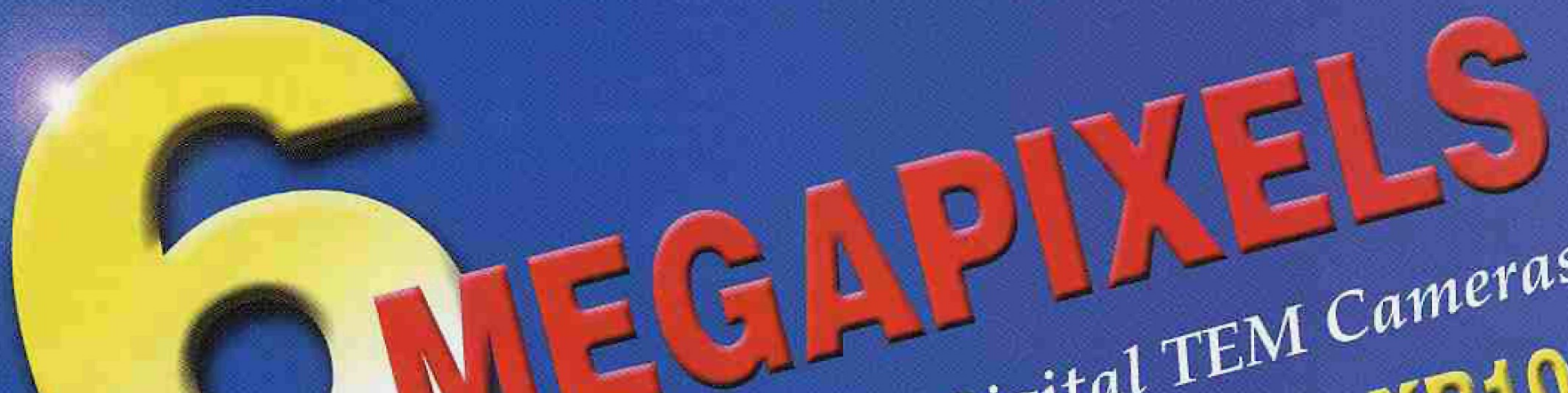
TEM
MTEQRATION

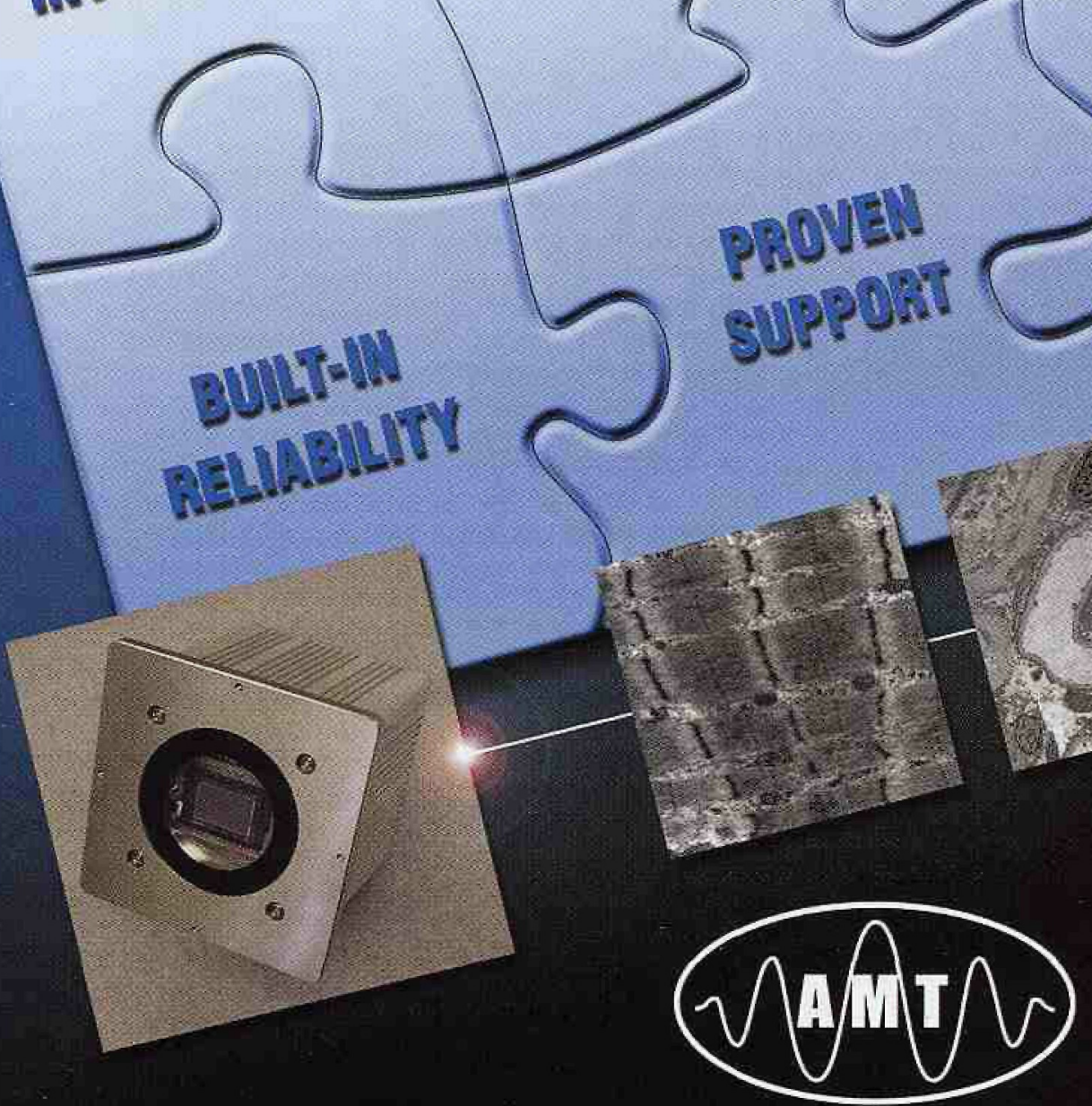

Advanced Mieroseopy Techninues Gorporation 3 Electronics Avenue • Danvers, ma 01923 978-774-5550 • wwwamtimaging.60m 


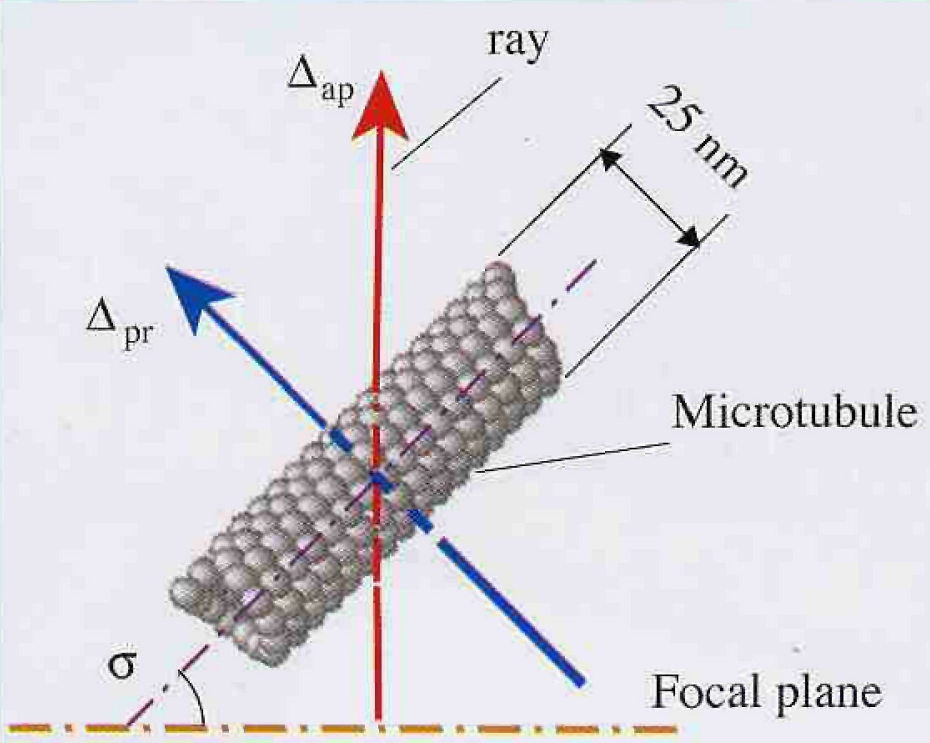

Fig. 2. Retardance of a microtubule inclined by an angle $\sigma$ to the objective focal plane. If the microtubule is illuminated with a cone of light that is oriented vertically (red arrow), an apparent retardance $\Delta_{a p}$ is measured that is smaller than the principal retardance $\Delta_{a p}$ of the microtubule. However, if the central ray of the illumination cone is tilted in the direction of the blue arrow, the measured retardance is equal to the principal retardance.

has correspondingly a higher retardance. The orientation of a microtubule in three dimensional space is described by 2 angles, the azimuth and the inclination. The azimuth is the orientation of the rod-axis in the focal plane of the microscope, the inclination is the angle by which the rod-axis is inclined to that plane. As mentioned earlier, with a regular polarizing microscope one can measure the azimuth, but not the inclination angle. Furthermore, as illustrated in Fig. 2, with a regular polarizing microscope one measures an apparent retardance, which varies with the inclination angle of the microtubule. Only if the inclination angle is zero does one measure the maximum retardance, which we choose to call principal retardance. Using the Scanned Aperture Pol-Scope, however, one can measure the principal retardance, the azimuth and inclination angle independently. Using traditional terminology, one might say that the Scanned Aperture Pol-Scope provides both orthoscopic and conoscopic measurements simultaneously for all birefringent objects in the field of view.

The Scanned Aperture Pol-Scope in our laboratory is implemented on an inverted microscope Zeiss Axiovert $200 \mathrm{M}$. The condenser mount was custom modified to add the scanning device in the aperture plane of the 1.4 NA oil immersion condenser lens. For recording the aster images shown next, we used a Plan-NEOUFLUAR $100 \times 11.30$ oil immersion objective, a high-pressure mercury arc lamp followed by an Ellis light scrambler (Technical Video, Woods Hole, MA) as the light source, and a narrow bandpass interference filter ( $546 \mathrm{~nm}, 12-\mathrm{nm}$ FWHM, Omega, Brattleboro, VT).

For demonstrating the workings of the Scanned Aperture Pol-Scope, we show images of an isolated aster, a spherically symmetric object consisting of microtubules radiating out in all directions from a centrosome. The overall shape of an isolated aster is similar to a kush ball that children toss and catch, except that the aster is only $20 \mu \mathrm{m}$ in diameter and is suspended in an aqueous medium. Fig. 3 shows images of the aster acquired with the Scanned Aperture Pol-Scope. The aster appears differently in the images depending on the configuration of the aperture mask and on the focus position of the objective lens. Because of the high numerical aperture of the objective lens, each image represents a thin optical section that is less than $0.5 \mu \mathrm{m}$ thick.

The images of astral microtubules in Fig. 3 represent the measured retardance of the MT arrays. For a given focus position and mask configuration, the retardance was measured using the universal compensator that is included in the aperture scanning device. Procedures for measuring the focus in center of aster
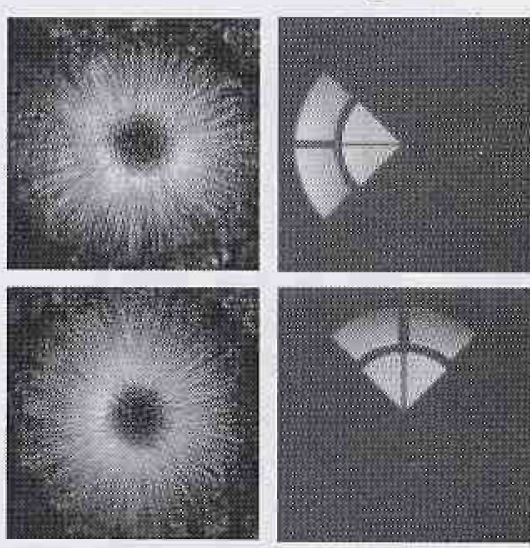

mask

focus $7 \mu \mathrm{m}$

from center
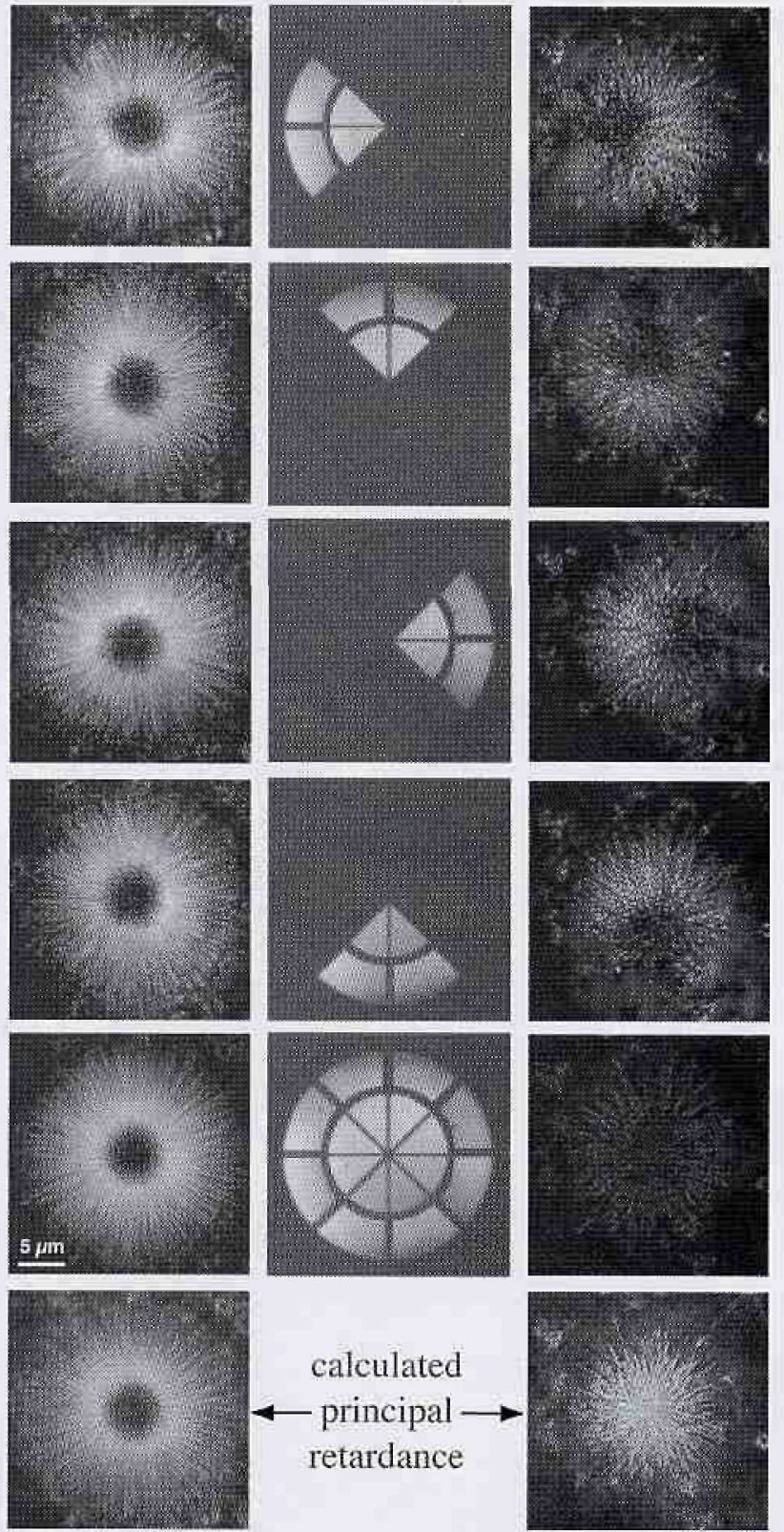

Fig. 3. Retardance images of astral microtubules recorded using various configurations of the aperture mask and two focus positions. Images in the left column represent thin optical sections that include the aster center. The aster center is formed by a centrosome, a non-birefingent protein structure that nucteates microtubules on its surface. Around the center, astral, microtubules are arranged radially with their long axis extending in the focal plane (inclination is zero). Images in the right column show astral microtubules extending through an optical section that is shifted by $7 \mu m$ from the center of the aster. Microtubules show varying degrees of inclination to the focal plane. As discussed in the text, for a given focus position, the astral microtubules change appearance depending on the inclination angle of the MTS and the aperture mask configuration. The central column shows the mask configurations as imaged using a Bertrand lens in the microscope optical train. The bottom row shows the calculated principal retardance maps, which are independent of MT ofientation and are directly proportional to the density of microtubules. We note that the aster images in the second row from the bottom ffully open aperture) correspond to images recorded with a regular polarizing microscope and should be compared to the bottom two images showing the principal retardance measured with the Scanned Aperture PolScope. (White corresponds to $3 \mathrm{~nm}$ retardance and biack indicates o nm retardance.) 


\section{STOP HASSLING WITH}

MULTIPLE SERVIGE CONTRAGTS!

START by putting all of your instruments under one service contract with MAS (regardless of make or model). Our expert EM SERVICE GROUP has the knowledge and skills to keep your instrument working at its best.

\begin{tabular}{|c|c|c|c|}
\hline \multicolumn{2}{|c|}{ TEMS / SEMS } & PREP EQUIPMENT & SPECIAL SERVIGES \\
\hline HITACHI & TOPCON & VACUUM COATERS & STAGES \\
JEOL & ISI & PLASMA ASHERS & BEAM BLANKERS \\
AMRAY CAMBRIDGE & SPUTTER COATERS & CUSTOM DEVICES \\
& ION MILLS & \\
\hline
\end{tabular}

\section{NEW NEW NEW}

Authorized service representatives for Gatan preparation equipment and Topcon TEM'S.
Contracts and On-Demand Emergency Service at

Reasonable Rates from Factory Trained Specialists.

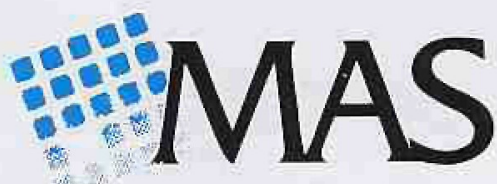

800-421-8451

3945 Lakeficld Court Suwance, Genrgin 30024 770-866-3200 FAX 770-866-3250 616 Hution Sinet Suite 101 Rileigh, North Carolina 23606 919-829-7041 FAX 919-829-5518 ADVANCED ANALYTICAL PRODUCTS AND SERVICES

\section{NORTHERN
- OCLIPSE}

LIMITED TIME OFFER

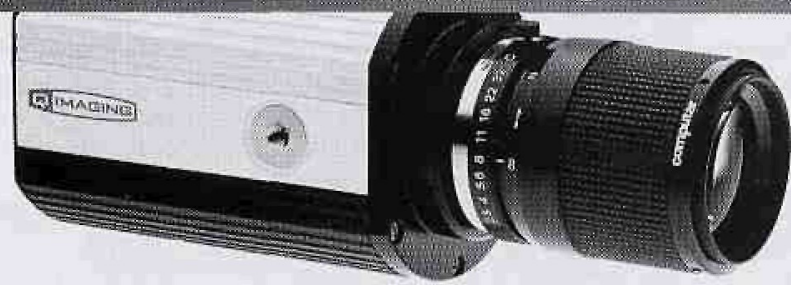

Get the power of Northern Eclipse 6.0 FREE by ordering a QImaging FireWire CCD camera When you buy a QImaging CCD FireWire camera directly from us at the manufacturer's suggested list price, we will include, for a limited time, one FREE Northern Eclipse 6.0 application of your choice. Applications such as Brightness/ Time, Time-Lapse, Image Merging, Fast Capture to Ram, and even De-convolution are available. See the On Sale page of our web site for a full list of applications available with this offer.

\section{www.empix.com}

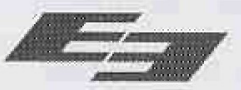

E M P |X

TM 'Firewire' is a registered trademark of Apple Computer Ino

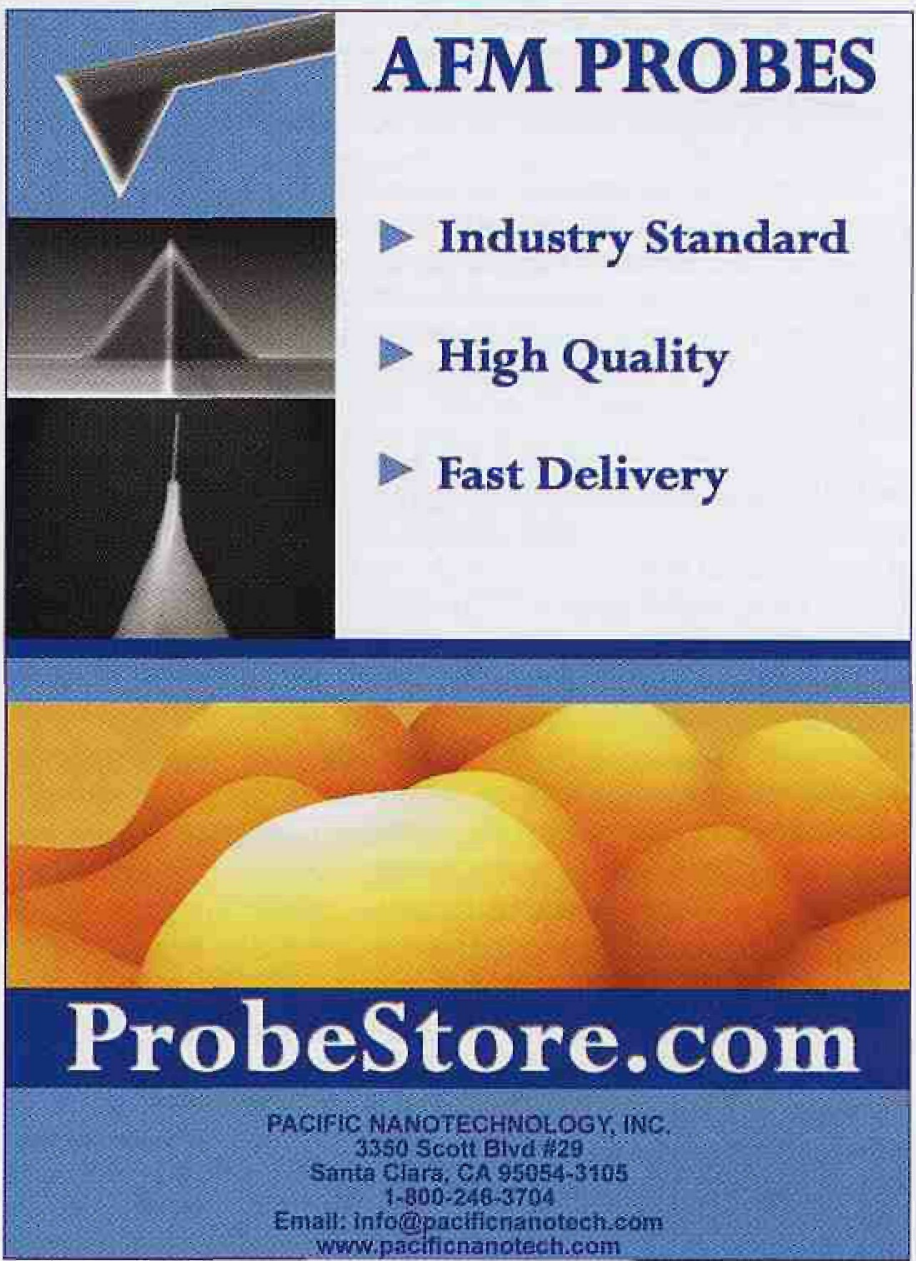




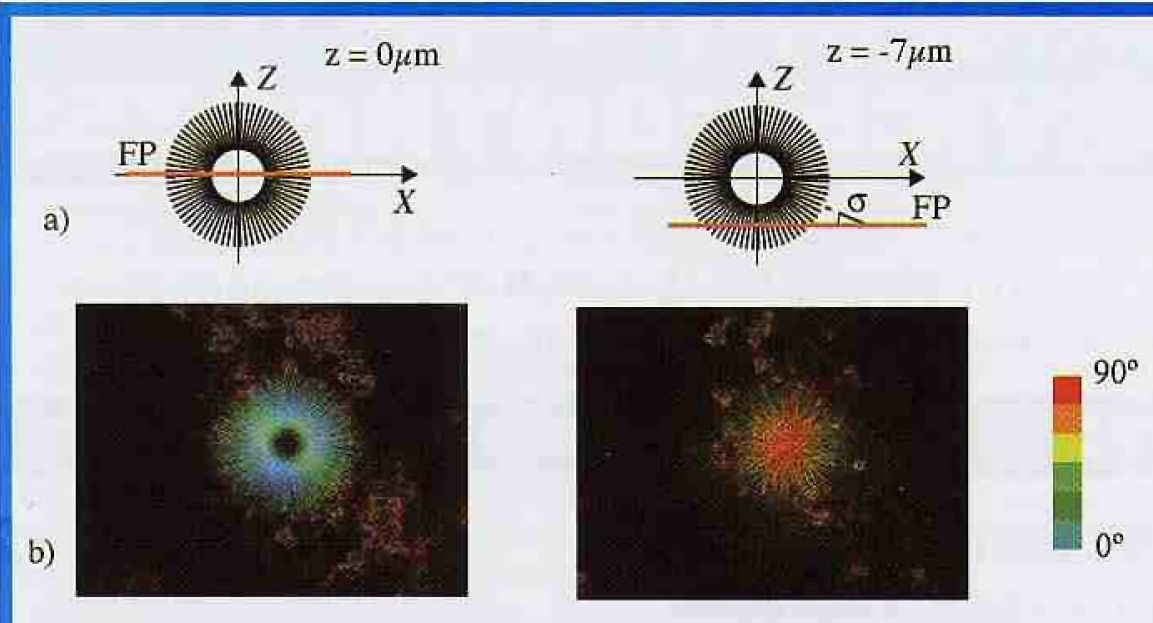

the observed microtubule arrays. Briefly, partial occlusion of the condenser aperture changes the direction of the central ray of the cone of light converging on the aster. Hence, depending on the mask configuration, a different set of microtubules extend their axis nearly parallel to the central ray and therefore appear black in the image. For example, the top right image of Fig. 3 was recorded with the left quarter of the condenser aperture open. Accordingly, the cone of light illuminating the aster is tilted to the left. As a consequence, the microtubule array imaged on the left side appears black because those MTs run nearly parallel to the central ray. However, the MTs in the middle and to the right of it appear brighter because their axis runs more perpendicular to the cone of light.

The appearance of astral MTs in the rest of the images in the right column of Fig. 3 change according to the position of the open aperture quarter. The fifth aster image from the top was recorded with a fully open aperture and, as expected, shows the central MTs black. The measured

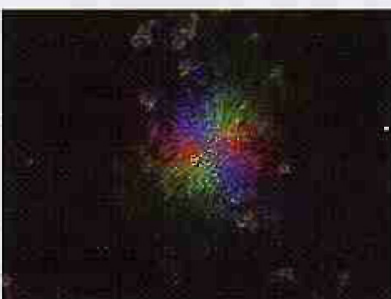

c)

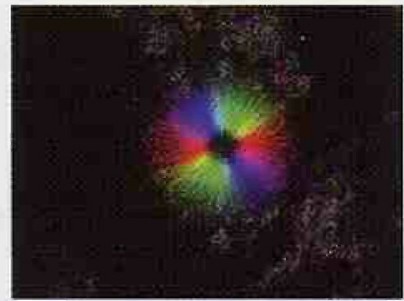

Fig. 4. (a) Schematics representing a side view of an aster. In this side view, the microscope axis (Z-axis) extends vertically, while optical sections (focal plane FP) are represented by horizontal lines. The two optical sections shown in Fig. 3 pass either through the center of the aster or are shifted by $7 \mu \mathrm{m}$ along the microscope axis; $\sigma$ is the inclination angle. (b) Images of the measured principal retardance and inclination angle for the two focus positions. The principal retardance is proportional to the brightness and color indicates the inclination angle. A color bar for the inclination angie is shown to the right. Both principal retardance and inclination angle were calculated based on the image data shown in Fig. 3. (c) Images of the measured principal retardance and the azimuth angle for the two focus positions. As in (b), principal retardance is proportional to the brightness. The color indicates the azimuth angle. A color bar for the azimuth angle is shown to the right.

apparent retardance and azimuth angle using the universal compensator are described in [3].

The left column of images in Fig. 3 shows thin optical sections through the center of the aster. The central part of the aster, out to a diameter of about $3 \mu \mathrm{m}$, is occupied by the centrosome that does not contain aligned microtubules and is not birefringent. Near the surface of the centrosome, at a diameter of about $3 \mu \mathrm{m}$, astral microtubules contained in central optical sections form a dense, radial array that extends parallel to the focus plane (inclination is zero).

The right column of images in Fig. 3 shows optical sections for which the focus was displaced $7 \mu \mathrm{m}$ from the center of the aster. Arrays of astral microtubules displayed in these sections are dense, even in the middle of the image, but their inclination angle changes. Near the middle of the section, the inclination angle is $90^{\circ}$ (parallel to microscope axis), while at increasing distance from the middle, the inclination angle of the microtubules decreases and becomes approximately $45^{\circ}$ near the outer edge of the aster (see aster schematics shown in top row of Fig. 4).

The central column of Fig. 3 shows images of the aperture mask that was used when recording the images in the column to the left and right. The mask images were taken with an additional Bertrand lens focusing on the aperture plane where the mask is located. For a given mask configuration, the corresponding retardance image of the aster is shown to the left and right of the mask image. As can be noted, images on the left, showing optical sections through the center of the aster, don't change much when the mask is changed. Images on the right, however, change dramatically when the mask configuration is changed.

The differences between the images in the right column can be understood on the basis of the systematic variation of the inclination angle of

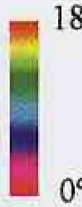

retardance in all five images were evaluated together using specifically designed algorithms to determine the principal retardance shown in the bottom image. As discussed earlier, the principal retardance is independent of the orientation and only depends on the density of the microtubules. As expected, the principal retardance has a maximum in the middle of the aster image where the density of aligned microtubules is the highest (see the aster schematics shown in the top row of Fig. 4). However, the high MT density is only expected if the focus is shifted away from the center of the aster. If the optical sections include the aster center, as is the case for the images in the left column of Fig. 3 , the principal retardance is zero in the center, because the centrosome is devoid of aligned microtubules and has no birefringence.

In addition to the principal retardance, the Scanned Aperture Pol-Scope can also measure the azimuth and inclination angle. Figure 4 shows color images in which color represents the measured inclination or azimuth orientation of the microtubules. The measured distributions of principal retardance, inclination and azimuth angles, do correspond closely to the expected structure of astral microtubules.

In summary, we have succeeded in developing a polarized light microscope for imaging birefringent object whose optic axis is inclined away from the focal plane, and measure the inclination angle and principal retardance of the object. This unique result is achieved with a scanned aperture polarizing microscope.

\section{References}

1. Oldenbourg, $\mathrm{R}$. and $\mathrm{G}$. Mei, New polarized light microscope with precision universal compensator. Journal of Microscopy, 1995. 180: p. 140-147.

2. Glazer, A.M., J.G. Lewls, and W. Kaminsky, An automatic optical imaging system for birefringent media. Proceedings of the Royal Society of London, Series A: Mathematical and Physical Sciences, 1996. 452: p. $2751-2765$

3. Shribak, M. and R. Oldenbourg, Techniques for fast and sensitive measurements of twodimensional birefringence distributions. Applied Optics, 2003. 42: p. 3009-3017

4. Shribak, M. and R. Oldenbourg. Sensitive measurements of two-dimensional birefingence distributions using near-circularly polarized beam. in Polarization Aralysis, Measurement and Remote Sensing V. 2002. Seattle: Proceedings of SPIE, 4819: p. 56-67.

5. Katoh, $K$, et al., Birefringence imaging direcily reveals architectural dynamics of filamentous actin in living growth cones. Molecular Biology of the Cell, 1999. 10: p. 197-210.

6. Keefe, D., et al, Imaging meiotic spindles by polarization light microscopy: principles and applications to IVF. Reprod Biomed Online, 2003. 7(1): p. 24-9.

7. Hartshorne, N.H. and A. Stuart, Practical Optical Crystailography. 1964, New York, NY American Elsevier Publishing Co., Inc.

8. Shribak, M. S. Inoué, and R. Oldenbourg, Polarization aberrations caused by differential transmission and phase shitt in high NA lenses: theory, measurement and rectification. Optical Engineering, 2002, 41(5): p. 943-954

9. Oldenbourg, R., E.D. Salmon, and P.T. Tran, Birefringence of single and bundled microtubules. Biophysical Joumal, 1998. 74: p. 645-654. 
Software to Make Your Job Easier, and a Free X-Checker Too!

\section{ANEW ALTERNATIVETOYOURTEM SPECIMEN HOLDER SERVICE AND REPAIR NEEDS.}

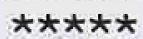

TGS Technologies Provides High Quality TEM Specimen Holder Service With 20 Years Experience.Inadditionweofferreasonablehourly serviceratesandmorepersonalizedservicefor you.

\section{Contact Us Today:}

TGS Technologies

Phone: 724.453.3865

E-mail: tom@tgstechnologies.net

Web: http://www.tgstechnologies, net

For a limited time, get a free $X$-Checker calibration/performance standard (a $\$ 250$ value) when you purchase Electron Flight Simulator.

Electron Flight Simulator became the world's leading SEM/X-ray visualization software by showing you how your sample behaves under all conditions

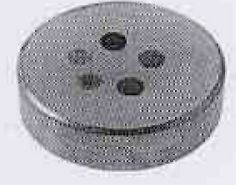

Put both to work in your lab and save $\$ 250$ !

www.small-world.net (703) 849-1492

Scalpels to Scoops to Screwdrivers to Spatulas to Speedles to Sputter Coaters Carbon Coaters to Clip Mounts to Carbon Rods to Custom Equipment Beakers to Beam Stops to Boats to Books to Bottles to Boxes Tape to Timers to Titanium Tweezers to Tensile Testers

Pipettes to Planchets to Pithwood to Power Supplies Hacksaws to Hex Grids to Heating Stages Universal Holders to Uranyl Acetate Vacuum Pumps to Viewing Boxes Wafer Tweezers to Work Holders Magnifiers to Micromanipulators Desiccators to Dropping Bottles Latex Spheres to Lens Tissue EFFA Dusters to Evaporators Glass Bottles to Grid Boxes Razor Blades to Ruby Mica Ferritin to Films to Forceps Acetone to Apertures What can you imagine? ERNEST F. FULDAM, INC.

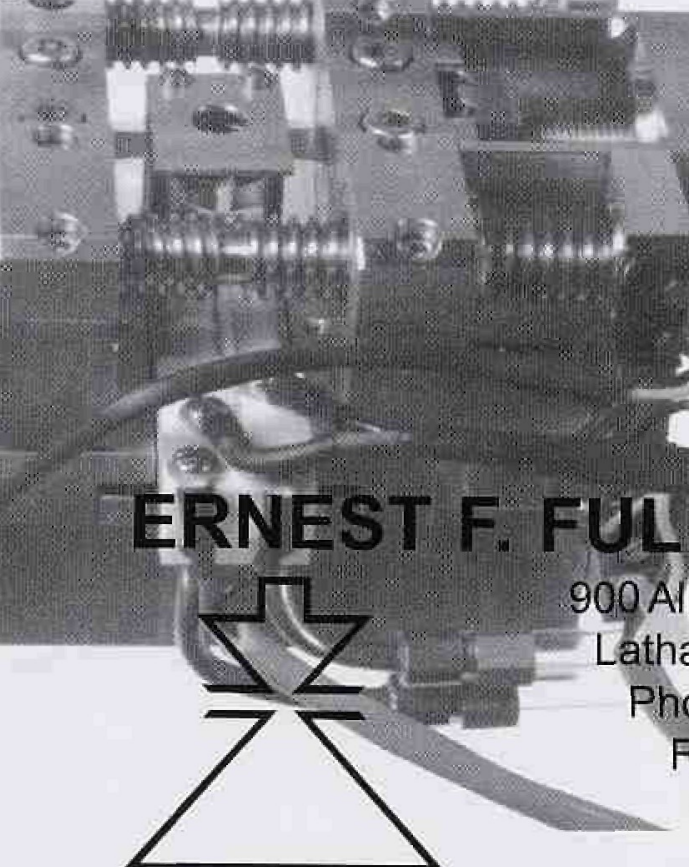

mIOROSCOPY TODAY November/December 2003
AX: $518785-8647$ sales@fullam.com www.fullam.com 